\title{
"Antes era más fácil". La incomodidad de enseñar Educación Física en tiempos de Educación Sexual Integral
}

\author{
"It used to be easier." The uneasiness of teaching Physical \\ Education in times of Comprehensive Sex Education
}

Jorgelina Andrea Marozzi ${ }^{1}$

\begin{abstract}
Resumen: La propuesta de la Educación Sexual Integral (ESI) promueve la revisión de prácticas de enseñanza tradicionales en el espacio curricular de Educación Física $(E F)$, lo cual genera dificultades en el colectivo docente, que debe revisar sus propuestas a la luz de nuevos paradigmas educativos.

Este artículo pone en evidencia modos de abordaje de la ESI y hace visibles supuestos subyacentes en el discurso del profesorado que obstaculizan implementar estrategias pedagógico-didácticas acordes con el enfoque de la ESI. Se exponen así las concepciones sobre el sentido de la EF en la escuela secundaria, la educación sexual, el género, entre otras.

Concluimos que la dificultad en la incorporación de la perspectiva propuesta por la ESI se sustenta en una serie de ideas y supuestos construidos según lógicas cientificistas, biologistas y deportivas de esta disciplina escolar, que requieren ser revisadas para apropiarse de nuevas perspectivas y prácticas.
\end{abstract}

Palabras clave: educación física, educación sexual, coeducación, práctica pedagógica.

\footnotetext{
${ }^{1}$ Magíster en Ciencias Sociales. Licenciada en Psicología. Profesora en Psicología. Docente de la Universidad Provincial de Córdoba. Integrante del Programa de Educación Sexual Integral del Ministerio de Educación de la provincia de Córdoba, Argentina. Correo electrónico: marozzijorgelina@gmail.com.
}

Diálogos Pedagágicas. ISSN en línea: 2524-9274.

Año XVIII, No 35, abril - septiembre 2020. Pág. 67-80.

DOI: http://dx.doi.org/10.22529/dp.2020.18(35)05 / Recibido: 28-07-2019 / Aprobado: 27-04-2020.

(c) (i) () $\odot$ Artículo publicado bajo Licencia Creative Commons Atribución-NoComercial-SinDerivar. (c) Universidad Católica de Córdoba. 


\begin{abstract}
The proposal of the Comprehensive Sex Education (CSE) promotes the revision of traditional teaching practices in the physical education curricular space (PE), which generates difficulties for the teaching staff, who must review their proposals in light of new educational paradigms.

This article highlights ways of approaching the CSE, and makes visible underlying assumptions in the teachers' discourse that hinder the implementation of pedagogicaldidactic strategies in accordance with the CSE approach. Concepts about the meaning of PE in secondary school, sex education, gender, among others, are exposed.

We conclude that the difficulty in incorporating the perspective proposed by the ISE is based on a series of ideas and assumptions construed under the scientific, biologist, and sports logic of this school discipline, which need to be revised to acquire new perspectives and practices.
\end{abstract}

Keywords: physical education, sex education, coeducation, teaching practice.

\title{
Introducción
}

La Educación Sexual Integral (ESI), instaurada como política de Estado en Argentina a partir de la Ley No 26.150 Programa Nacional de Educación Sexual Integral, instituye la responsabilidad de escuelas y docentes de garantizar el derecho a una educación sexual integral a todo el estudiantado. En este marco, el cuerpo docente de Educación Física no está exento de esta tarea. Para el espacio curricular de Educación Física, las sugerencias de implementación desde las políticas ministeriales apuntan, por un lado, a modificaciones en los modos de organización o agrupamiento de los y las estudiantes e instan al trabajo en grupos mixtos y, por otro lado, recomiendan modificaciones curriculares en las cuales se incluyan enfoques y contenidos que históricamente ocuparon lugares periféricos en los programas escolares.

Teniendo en cuenta que se trata de una política educativa relativamente reciente, son escasas las investigaciones que indagan acerca de la implementación de la ESI en el espacio curricular de la Educación Física $(E F)^{2}$. En Córdoba, un equipo de investigación, dirigido por la autora del presente artículo, recuperó los discursos que sostiene el profesorado de EF en torno a la implementación de la $E S^{3}$ y, a través de entrevistas y análisis de discusiones docentes, identificó algunas resistencias que están operando en la modificación de los agrupamientos tradicionalmente separados por sexo a agrupamientos mixtos (para profundizar acerca de estos resultados, consultar Marozzi, Bosio \& Bertarelli, 2018).

\footnotetext{
2 En contraposición, sí son abundantes los trabajos que indagan la receptividad del enfoque de género en este campo curricular. Para ampliar sobre el estado de la cuestión, ver Kopelovich y Pansa, 2017.

3 Proyecto de Investigación "El posicionamiento docente frente a la implementación de la Educación Sexual Integral en Educación Física de la escuela secundaria", Marozzi, J. (directora); Bertarelli, P. Boccardi, F., Bosio M.; Raviolo, A.; Videla, S. UPC 2017-2019. Aprobado y financiado por el Ministerio de Ciencia y Tecnología Córdoba.
} 
Otro núcleo de resistencias se concentra en torno a las modificaciones en la enseñanza. En el marco de la investigación mencionada anteriormente, el presente artículo desarrolla este eje de resistencias y muestra cómo interpreta o entiende el profesorado que debe abordarse la ESI en la escuela y, específicamente, desde su espacio curricular. Se hacen visibles desde qué ideas de masculinidad, feminidad, género, entre otras, se están interpretando las modificaciones curriculares que propone la ESI. El artículo también avanza en consideraciones respecto a qué concepciones de $\mathrm{EF}$ se ponen en juego al implementar (o resistirse) a la ESI. En el texto, solo se incluyen algunos testimonios con el objetivo de ilustrar y desmenuzar algunos enunciados que se repiten en el discurso de los participantes de la investigación arriba mencionada.

\section{Contextualización de la problemática: Género, ESI y EF}

Si rastreamos la matriz constitutiva de la EF a fines del siglo XIX y su desarrollo como disciplina escolar a lo largo del siglo $\mathrm{XX}$, nos encontramos con prácticas atravesadas por discursos militaristas, higienistas, tensionados por discursos pedagógicos, y por la incorporación de la fuerte presencia de juegos y deportes que van imprimiendo rasgos particulares según sea la época en la cual focalicemos la mirada. Pero lo cierto es que, cualquiera sea el período de tiempo que consideremos, estos discursos provenientes de diferentes lugares de enunciación siempre son generizados, en el sentido que prescriben -con mayor o menor fuerza- contenidos y actividades diferenciales para varones y mujeres.

La separación de mujeres y varones para la enseñanza de la disciplina respondió, en un primer momento, a supuestos morales asociados a la masculinidad (osadía, fuerza, valor, coraje) y a la feminidad (decoro, gracia, elegancia, delicadeza) que la educación física podría ayudar a desarrollar (Scharagrodsky, 2006). Cuando en las décadas de los sesenta y setenta del siglo pasado se comienza a cuestionar la visión disciplinante y excluyente en relación al género, dicha separación continuó, ahora sostenida en discursos médicos y fisiológicos que, desde una visión esencialista de los cuerpos, atribuyen diferencias físicas entre varones y mujeres.

Los enfoques críticos mostraron de qué manera la EF y el deporte históricamente desempeñaron un rol en favor de un poder hegemónico que buscó la disciplina de los cuerpos e instaron a elaborar propuestas que contemplen la importancia del juego y la expresión, el placer y el disfrute del tiempo libre en contacto con la naturaleza (Kirk, 1990; Bracht, 1996; Martínez \& Gómez, 2009). Por otra parte, los desarrollos de los estudios de género vinieron a develar que no existe una esencia femenina o masculina determinada por la naturaleza, sino que estas son construcciones sociales que se realizan sobre la base de la diferencia sexual, que -además- es interpretada binariamente (De Lauretis, 1996; Lamas, 1996; Butler, 2001). Estos estudios permean el campo de la educación física, cuestionan que las diferencias actitudinales y de comportamientos de los sujetos sean inherentes a su constitución física y problematizan, por lo tanto, la enseñanza diferenciada entre varones y mujeres que refuerza estigmatizaciones y desigualdades, 
para apostar al trabajo conjunto. Esta discusión se dio con fuerza en España, en las décadas de los ochenta y noventa del siglo anterior, al nuclear en el concepto de coeducación la necesidad de brindar igualdad de oportunidades a varones y mujeres en las prácticas educativas, atender a la revisión de pautas sexistas, reducir la desigualdad y la jerarquía de lo masculino, tendiente a que cada uno pueda desarrollar sus cualidades individuales.

Estos debates ingresan en Argentina cada vez con mayor fuerza. Son pioneras en nuestro país las reflexiones de Jorge Saraví (1995), que giran en torno a cuestionar los modelos estereotipados y sexistas que dominan en la enseñanza de la disciplina separada por sexos. En esta línea, Pablo Scharagrodsky (1999) viene a inaugurar una serie de trabajos investigativos que observan las clases de EF en clave de género. Kopelovich y Pansa (2017) realizan un estado de la cuestión sobre EF, géneros y sexualidades en Argentina y muestran la proliferación de trabajos desarrollados en las últimas dos décadas, que revisan la historia de la disciplina, la formación docente, el currículum y la didáctica de la EF al poner en juego los aportes del enfoque de género $y$, específicamente, cuestionan la organización separada de las clases. No obstante, estas investigaciones en el ámbito académico y su incorporación en los currículos de las carreras de formación docente no impregnaron la práctica cotidiana de las escuelas, que continúa rigiéndose por las tradiciones de enseñanza (Di Gregorio, 2013; Kopelovich, 2017; Pellegrini Malpiedi, 2015).

Con la sanción de la Ley 26.150 en 2006, la propuesta de la ESI actualiza una discusión que no es nueva en el campo de la EF, pero que cobra fuerza al legitimar nuevos saberes y prácticas. La ESI, sustentada en la perspectiva de género y derechos, reclama con fuerza la igualdad de oportunidades entre varones y mujeres. Esto se plasma en los Lineamientos Curriculares para la Educación Sexual Integral (Argentina, Ministerio de Educación, 2008) que enfatizan la importancia que adquiere "la comunicación corporal entre varones y mujeres" (p. 39) en la práctica de actividades físicas. La ESI también considera "el respeto por la diversidad de identidades y de posibilidades motrices, lúdicas y deportivas" ( $p .39$ ) e insta al desarrollo de prácticas inclusivas y no discriminatorias. Además, tiene en cuenta la necesidad de reconocer y expresar emociones y sentires que contribuyan a generar espacios que promuevan la convivencia, participación y cooperación por sobre la competitividad.

Ante esto, una parte del profesorado expresa resistencias y dificultades para la implementación. ¿Qué sustenta dichas resistencias? ¿Desde qué estructura de recepción (Giordan \& De Vecchi, 1988) los y las docentes están interpretando el abordaje de la ESI? ¿Qué ideas de cuerpo sostienen? ¿Qué piensan sobre la construcción de la feminidad y la masculinidad? ¿Para qué creen que es necesario la EF en el nivel secundario? En síntesis, ¿cuál es el marco de referencia desde el cual se apropian (o no) de la ESI? Partimos de considerar que estas ideas están sosteniendo sus modos particulares de enseñanza y, por ello, se hace necesario conocerlos e interpelarlos. 


\section{Los "malabares" para enseñar educación sexual}

Son diferentes las maneras en que el profesorado puede manifestar su disconformidad en torno a la implementación de la ESI tal cual es requerida desde los organismos ministeriales a través de supervisores y supervisoras, equipos técnicos, directivos. Una de ellas es hacerlo explícitamente a través de notas a superiores y declaraciones públicas en medios de comunicación. Otra manera, si se quiere más implícita, pero también más efectiva, es no producir ninguna modificación de las requeridas: sin clases mixtas ni revisión de los contenidos ni estrategias metodológicas.

La falta de modificación de las propuestas ¿se debe a un desconocimiento de la ESI? ¿No acuerdan con la perspectiva que la sostiene? ¿Cómo entienden los y las docentes que pueden abordar la ESI desde este espacio específico? Veamos algunos fragmentos del discurso de una docente ${ }^{4}$ que relaciona la ESI con "hablar sobre los cambios adolescentes":

"Digo cambios adolescentes a ver 'vengan los varones para acá'. Las chicas estaban jugando al hándbol, en ese momento los dividí porque tenía que hablar de sus cambios, entre ellos, no frente a las chicas en ese momento, entonces hablé con ellos mientras las chicas jugaban. Y después hice al revés, hablé con las chicas mientras los varones jugaban. Tuve que hacer esos malabares". (Nélida)

Este discurso, reforzado por la docente en otros momentos de la entrevista al reproducir el diálogo entablado con sus estudiantes, da cuenta de un escaso conocimiento del enfoque integral de la educación sexual, que queda reducida a "hablar" de aspectos anatómicos y funcionales del sistema sexual y reproductivo. Este enfoque biologista (Morgade \& Alonso, 2008) primó en las tradiciones de enseñanza en las escuelas y a él se opone el enfoque integral que se sostiene en una concepción amplia de sexualidad, "resultado de la interacción de factores biológicos, psicológicos, socioeconómicos, culturales, éticos y religiosos o espirituales" (Organización Panamericana de la Salud, Organización Mundial de la Salud, 2000, p. 6). El enfoque integral pone el acento en recuperar y problematizar la (re)producción de estereotipos y prácticas sobre las sexualidades y géneros y sus efectos en las subjetividades, a la vez que propicia relaciones de género equitativas, en un marco de respeto a los derechos de todos y todas. Favorece la expresión de emociones y manifestaciones afectivas que intervienen en los modos de vivir, cuidar, disfrutar, vincularse con otros y otras para el desarrollo de una sexualidad placentera y responsable (Marina et al., 2014).

Es interesante remarcar que la propuesta de enseñanza mixta que la ESI promueve como modo de instar a la igualdad de oportunidades es interpretada por la docente como un obstáculo ante el cual tiene que hacer "malabares" para enseñar lo que ella considera educación sexual.

${ }^{4}$ A lo largo del artículo, se recuperan fragmentos del registro de entrevistas realizadas a docentes participantes de la investigación antes mencionada. Se entrevistó un total de siete docentes en ejercicio, que habían manifestado públicamente su oposición a las modificaciones organizacionales y didácticas que propone la ESI a través de notas presentadas a directivos de sus escuelas e inspectores. Los nombres están cambiados. 
Por otra parte, si hay desconocimiento del enfoque, ¿desde dónde se responde? Al preguntarle a Nélida por qué separaba a varones y mujeres para hablar de cambios adolescentes, nos explicita:

"Porque yo, un médico el Dr. Brone (inentendible) en el colegio Juan Luis Gonzaga hace muchos años atrás, estábamos en segundo año, éramos todas chicas [...] y ahí nos habló de la menstruación, de las hormonas, del aparato reproductor, esto lo otro, y siempre tiene que haber un mínimo, aunque sea en la primera conversación, un mínimo de privacidad".

La docente apela a sus recuerdos de adolescente, a los modos en que recibió educación sexual para abordar ahora esta temática con sus estudiantes. La historia personal se constituye en la fuente de conocimientos $y$, por lo tanto, el enfoque biologicista no es interpelado por nuevos saberes. Si para la docente la sexualidad queda reducida a su dimensión biológica, podemos pensar que la Educación Física aportaría a su enseñanza en tanto se la conciba como una educación para la salud. El objetivo de favorecer el desarrollo de la salud y el mejoramiento de funciones básicas ligadas al cuerpo a través del movimiento y la actividad física es uno de los mandatos tradicionales de la asignatura que las nuevas perspectivas en la disciplina vienen a revisar. Este enfoque tradicional de la EF puede observarse durante todo el discurso de la docente en enunciados como "el currículum de Córdoba es muy amplio, pero le falta un poquito de la parte de nutrición, sería una carencia que me parece esencial porque está muy relacionado con el movimiento y con la salud" o "muchas enfermedades son provocadas por el sedentarismo, o sea que nuestra materia tiene una importancia fundamental en la salud futura de los chicos". Si para la docente este es el aporte que hace la EF a los y las jóvenes en la escuela secundaria, es esperable que la educación sexual que implementa quede reducida a aquellos contenidos que contribuyen al conocimiento del cuerpo entendido solamente en su dimensión biológica.

Por el contrario, en el Diseño Curricular Ciclo Básico de Educación Secundaria vigente en la provincia de Córdoba (Córdoba, Argentina. Ministerio de Educación de la Provincia de Córdoba, 2011), la EF es concebida como práctica social que "interviene para la apropiación y recreación de saberes propios de la cultura corporal" (p. 144) e insta a que las prácticas de enseñanza tomen en cuenta la biografía corporal de cada joven y habiliten espacios para sus singulares modos de expresión motriz. Podemos pensar que subyace así una concepción de cuerpo inserto en una trama de sentido y significación, atravesado por la cultura. Lejos de concebirlo como conjunto de huesos, articulaciones y músculos, se lo piensa como "terreno de disputa en el que se aloja un conjunto de sistemas simbólicos entre los que se destacan cuestiones vinculadas al género, a la orientación sexual, a la clase, a la etnia o a la religión" (Scharagrodsky, 2007, p. 2).

Veamos el discurso de otro docente:

"Desde la dirección se armó un cronograma que participaban profes de distintas áreas, a mí me tocó con historia y geografía armar un proyecto de ESI [...] Primero teníamos que hacer cada profe solo, entonces yo busqué dos videos de diversidad y discriminación que eran muy didácticos [...] hice la actividad en un día, tenían que traer un afiches, fibrones, y yo les tiraba una palabra a cada grupo, transexual, 
homosexual, bisexual, y cada grupito ponga un concepto de esa palabra, de lo que entienda, y después fuimos a ver el video [...] después tenían que traer una figura del diario, revista o dibujar lo que le había tocado y pegar en el afiche". (Marcelo)

Lo primero que aparece es la percepción de la ESI como un contenido desvinculado de la actividad corporal y motriz propia de este espacio. El docente planifica actividades donde el protagonismo corporal no está presente, al punto tal que pareciera que hay que salirse de lo disciplinar para dar educación sexual. Más que pensar a la ESI como una perspectiva que atraviesa toda la clase, se la percibe solo vinculada a contenidos específicos a ser abordados con didácticas más emparentadas con otros campos de conocimiento.

En los documentos ministeriales elaborados para favorecer el abordaje de la ESI en la EF, son numerosas las referencias a la posibilidad de implementar múltiples prácticas motrices, lúdicas, expresivas y deportivas a través de las cuales se pongan en juego contenidos y aprendizajes ligados a la sexualidad.

Por otra parte, algunos y algunas docentes entienden que los aprendizajes propuestos por la ESI se adquieren a partir de las actividades motrices, pero subyace la creencia de que por el mero hecho de unir varones y mujeres ya es suficiente y no requeriría otra modificación más que el reagrupamiento en clases mixtas. Podemos inteligir esta concepción en el enunciado de este entrevistado:

"Trabajamos sin querer la ESI nada más que no lo ponemos en el libro de tema, si yo me pongo a trabajar con vos en vóley estoy haciendo una relación con vos, didáctica, de ensayo y error, de correcciones, y yo estoy aceptando que vos tenés limitaciones o yo tengo limitaciones. Por ahí tengo una chica que tiene mucha habilidad para el deporte y un varón que no, y bueno, se complementan, y hay una relación ahí". (Marcelo)

Si bien el agrupamiento mixto es condición indispensable para la ESI, no es suficiente si el o la docente no hace otras mediaciones que apunten a un trabajo coeducativo, donde se intervenga ante la reproducción de prácticas sexistas, se elimine la jerarquía de lo masculino que suele primar en las interacciones, se contemplen las diferencias, se reduzcan las desigualdades y se potencien las cualidades de cada estudiante a partir de una real igualdad de oportunidades.

La reorganización de grupos tradicionalmente separados por sexo en grupos mixtos no significó necesariamente, para el colectivo docente, la modificación de sus propuestas didácticas, sino que para algunos y algunas docentes implicó la extensión de las propuestas tradicionales a todo el estudiantado.

Vemos así cómo el discurso de estos educadores evidencia un desconocimiento tanto del enfoque como del modo de implementación de la ESI y sostiene propuestas que se alejan del abordaje integral que la educación sexual demanda.

\section{La implementación de la ESI: intervenciones docentes}

Las clases mixtas trajeron consigo, para muchos integrantes del cuerpo docente, cierta incomodidad. El profesorado coincide en que "antes era más fácil dar 
la clase", ya que el interés de los y las estudiantes estaba más garantizado. "Antes [se refiere a la enseñanza por escuadras] le dábamos la oportunidad a los chicos que eligieran lo que les guste para trabajar, entonces nos garantizábamos que ese chico iba a trabajar, porque le gustaba eso" (Marcelo). Si bien, tal como desarrollamos en el apartado anterior, la reunión de varones y mujeres en un mismo tiempo y espacio no alteró demasiado las propuestas pedagógicas de algunos y algunas docentes, a otros les generó la necesidad de repensar las estrategias metodológicas que venían utilizando, tal como presentaremos a continuación.

Podemos distinguir dos tipos diferentes de acciones o intervenciones que pone en juego intencionalmente el colectivo docente: por un lado, adaptaciones a las actividades y, por otro lado, la introducción de nuevas actividades corporales. Dichas intervenciones están imbuidas de creencias acerca de la masculinidad y la feminidad, del cuerpo, la educación física, que se hace necesario desentrañar.

\section{Adaptaciones en los juegos, deportes y ejercicios motores}

En el nivel secundario, las prácticas deportivas adquieren centralidad. Cuando los y las docentes de este estudio rememoran sus clases, suelen referirse a prácticas deportivas; el fútbol, el hándbol, el vóley son los deportes predominantes. Si tenemos en cuenta que, en nuestra sociedad, el deporte tradicionalmente fue practicado por varones, quienes -desde muy pequeños- son alentados a estas prácticas, fácilmente podemos ver que el colectivo de mujeres, con algunas excepciones, no suele contar con el desarrollo corporal que esta práctica estimula y queda en desventaja cuando la propuesta docente no considera esta desigualdad.

Los y las docentes manifiestan: "Yo incluyo, pero si no quiere jugar al fútbol que juegue a la soga, al quemado, que se siente" (Andrea). "La chica que quiere jugar se tiene que adecuar y aguantar y soportar" (Graciela). "Los varones quieren jugar al fútbol, y algunas chicas se adaptan, pero otras no y se apartan" (Marcelo). Vemos en estos enunciados que son "las chicas" las que deben adaptarse a una propuesta tradicionalmente pensada para varones.

Quienes realizan adaptaciones en sus clases nos dicen: "Los varones tiran con la mano inhábil al arco si hay una mujer, para que tire menos fuerte y para que haga una resolución de problemas con el lado no hábil [...] Si hay un arquero varón, el varón tira libre" (Marcelo). "No hacen el gol si no se la pasan a una chica" (Graciela). "Hacemos un circuito los varones hacen diez flexiones de brazos y las chicas hacen cinco, con rodillas apoyadas que es menos peso" (Marcelo).

Las adaptaciones se realizan sobre las reglas de los juegos o deportes que se están enseñando o en la cantidad de ejercicios solicitados y siempre implica limitar las habilidades masculinas, que se consideran superiores, y obligar o alentar la participación femenina, ya que esta no estaría garantizada. La adaptación se hace conservando el mismo modelo de clase en el cual el énfasis está puesto en la adquisición de técnicas para aumentar el rendimiento. 
Estas estrategias se apoyan en una concepción naturalista de los cuerpos, ya que se prescriben de antemano actividades diferenciadas a cada colectivo (mujeres-varones) sin tener en cuenta las posibilidades reales de cada uno. Los varones, por el solo hecho de serlo, deben limitar su capacidad ("tirar con la mano inhábil") o demostrar su resistencia haciendo más ejercicios. Las diferencias entre los sujetos son leídas dicotómicamente $y$, en esa lectura, funcionan estereotipos acerca de las capacidades y habilidades de las mujeres y varones.

Es curioso que, durante las entrevistas, cuando se repregunta si existen mujeres que realizan ejercicios con la misma exigencia de los varones, y varones que no logran la meta fijada para ellos, el profesorado reconoce esto; sin embargo, al proponer consignas diferenciadas, lo hacen desde sus representaciones previas imbuidas de estereotipos.

La adaptación a la regla respecto a "pasar la pelota a una chica" antes de hacer un gol o "el gol vale doble" si lo realiza una chica suele ser una consigna recurrente en el intento de lograr mayor equidad, ya que se consideran las desigualdades que suelen funcionar cuando se realizan deportes tradicionalmente masculinos. No obstante, ¿qué mensajes implícitos se filtran junto a esas consignas? El mensaje refuerza la idea de debilidad o inferioridad de las mujeres por el solo hecho de ser mujeres y obtura la posibilidad de introducir momentos de reflexión con los y las estudiantes para problematizar las relaciones de poder que se visualizan durante las actividades conjuntas.

\section{Introducción de nuevas actividades}

Parte del profesorado reconoce que es en la práctica de deportes en la escuela, especialmente deportes hegemónicos, donde se concentran las mayores dificultades para el trabajo en grupos mixtos. En nuestro país, la oportunidad para acceder a prácticas deportivas es desigual, tal como señalamos previamente, y el colectivo de varones es quien -en mayor medida- accede a ellos durante la niñez. Esto explica las diferencias en relación al punto de partida de muchas jóvenes al encontrarse, por primera vez, ante esta práctica en el nivel secundario. Sumado a esto, la existencia de prácticas sexistas que se reproducen durante el juego (Fernández, 2011; Scharagrodsky, 2004) genera que la clase de Educación Física se transforme en un espacio reproductor de desigualdad.

Considerando esto, hay docentes que proponen la introducción de deportes alternativos, como modo de equilibrar el punto de partida y disminuir las desigualdades. Los deportes alternativos ofrecen la posibilidad de aprendizaje de múltiples valores a través del trabajo colaborativo.

Otra estrategia que implementa el profesorado es incluir -en mayor medidaactividades recreativas, con el fin de disminuir aquellas que generan competencia entre estudiantes. Parecería que, cuando la competencia es un aspecto central en la propuesta docente, las dificultades se exacerban. Por el contrario, cuando las actividades recreativas cobran protagonismo, el trabajo conjunto y los aprendizajes vinculados al placer en el encuentro con el otro se facilitan. Sin embargo, estas 
actividades suelen ocupar un pequeño porcentaje de tiempo durante las clases o durante el año. Nos dice Mariela: "Las primeras clases del año, cinco o seis clases hacemos todo recreativo, juegos y ahí se prenden la mayoría".

Una adaptación curiosa es aquella que lleva a incluir más actividades que requieren realización individual, como los circuitos funcionales, con el objetivo de reducir las posibilidades de trabajo conjunto. Andrea relata: "Para que no sea tan problemático para mí hago circuito funcional, que es lo que está ahora si van a un gimnasio es lo que se está dando". Ante esto, cabe preguntarse: ¿cómo desarrollar la comunicación corporal entre mujeres y varones que la ESI propone si se busca intencionalmente el trabajo individual? ¿Cómo posibilitar una clase coeducativa si se evita el contacto?

Teniendo en cuenta que del universo de prácticas corporales solo se han aprendido algunas alternativas en el ámbito escolar y se han excluido otras posibles, la estrategia de incorporar nuevas actividades comienza a acercarse a lo que propone la ESI. Sin embargo, se siguen pensando actividades bajo la lógica de enfoques tradicionales de enseñanza. Las nuevas propuestas no incorporan otras manifestaciones de la cultura corporal como la danza o la expresión corporal. "La murga, el hip-hop, las actividades circenses [...] no solo son expresiones interesantes, sino que esencialmente son manifestaciones de la cultura que se constituyen en excelentes recursos para colocar en un lugar privilegiado la expresión de las emociones y los pensamientos" (Argentina, Ministerio de Educación de la Nación, 2012, p. 46). Incluir este tipo de propuestas implica un cambio radical en los modos tradicionales en que se ha pensado la EF, cuyos mandatos fueron definiendo una mirada científico-técnica de los movimientos corporales que se tradujo en propuestas reproductoras y eficientistas. La ESI reclama, en coherencia con las nuevas perspectivas en $\mathrm{EF}$, dar lugar a otros escenarios motrices, lúdicos y deportivos que den respuesta a intereses diversos, que pongan el acento en el hacer y el sentir de los movimientos, que contribuyan a la desnaturalización de ideas estereotipadas respecto al cuerpo y al género y que aporten a la construcción de la identidad personal.

\section{Conclusiones}

Hicimos un recorrido por diversos modos de intervenir en las clases de EF que los y las docentes de este estudio ponen en juego para abordar la educación sexual integral. Observamos un desconocimiento del enfoque y modos de abordaje de la ESI, la cual queda reducida a la enseñanza de aspectos biológicos y con metodologías que no contemplan el compromiso corporal propio de esta asignatura. Se visualiza a la ESI como un contenido específico a trabajar en un momento determinado y no como un enfoque que atraviesa cada propuesta pedagógica.

La reorganización en grupos mixtos que esta política viene a proponer no implicó necesariamente el desarrollo de nuevas prácticas de enseñanza que contemplen la diversidad de género, que desnaturalicen ideas estereotipadas respecto al cuerpo, disminuyan la desigualdad, favorezcan la expresión de emociones e incorporen la dimensión de lo placentero. Por el contrario, se siguen soste- 
niendo propuestas tradicionales que, en esta nueva organización de grupos, aumentan las dificultades de enseñanza y aprendizaje. En los casos en los que se intentan modificar las propuestas, en general, no superan los formatos tradicionales.

Transversalizar la ESI en las propuestas de enseñanza requiere interpelar el orden escolar y, así, a todas las dimensiones prácticas educativas (los contenidos, las relaciones, las normas, etc.). En este sentido, implica una "crítica epistemológica a los saberes de referencia de los contenidos escolares y de las relaciones pedagógicas cotidianas" (Colectivo Mariposas Mirabal, 2019, p. 42). Supone también, en el mismo acto, la adopción de un posicionamiento pedagógico político sustentado en un enfoque de género y derechos, plataforma fundamental de la ESI.

Consideramos que la particular resistencia que manifiestan los y las docentes a la incorporación del enfoque de ESI en sus propuestas de enseñanza está condicionada por el posicionamiento sostenido en torno a la Educación Física. Si bien parte del profesorado puede no haber accedido a la formación específica en ESI, los enunciados dan cuenta de una plataforma de saberes ligados a perspectivas tradicionales de la EF que obturan la recepción del enfoque integral de la educación sexual.

Reconocemos que un gran porcentaje de docentes que hoy se desempeñan en las escuelas (y que constituyeron la población de la investigación en la que se basó el presente artículo) transitaron su formación hace más de quince años. Podemos hipotetizar que dicha formación estuvo marcada por enfoques tecnicistas y biologicistas de la EF (Bracht \& Crisonio, 2003), arraigados en disciplinas o áreas de conocimiento cuyo interés no radica en la intervención educativa, y con un predominio de la enseñanza de la técnica de los deportes.

Parecería que los enfoques críticos en la enseñanza de la disciplina no impactaron en la formación continua de estos profesores en ejercicio. Ariel, uno de los entrevistados, nos proporciona una explicación. Refiere que hay profesores que "vienen desde los veinte años dando de la misma forma y no sé si tienen ganas de cambiar [...] hay profes grandes que ya tienen una estructura formada porque a ellos también los formaron en el profesorado con esa estructura entonces uno va reproduciendo".

Podemos pensar que imperan, en el discurso de docentes, mandatos ligados a las matrices de origen de este campo disciplinar. Las visiones biologicistas, deportivas y tecnicistas dificultan apropiarse de nuevas perspectivas y prácticas. La dificultad de elaborar acciones acordes a las particularidades del grupo de clase se sostiene en una concepción naturalista de los cuerpos que son pensados solo en sus aspectos biológicos y no en su atravesamiento social y cultural. Además, impera una visión esencialista de la sexualidad y del género, que considera a la masculinidad y a la feminidad como determinadas por la naturaleza y no como construcciones culturales.

La propuesta de la ESI, así como la realidad escolar, vienen a cuestionar ideas y supuestos en los que se ha asentado esta disciplina, lo cual requiere docentes que hayan atravesado un proceso de revisión de estas concepciones tradiciona- 
les. Requiere, también, que hayan incorporado, en su formación inicial y/o continua, enfoques críticos que les permitan diferenciar el campo disciplinar de otros campos de los cuales históricamente extrapolaron muchos de sus postulados (campo militar, sanitario, deportivo) para inscribirlo con fuerza en el campo educativo y dotar así de sentido pedagógico a la EF en la escuela secundaria. Tal vez así la incomodidad manifestada pueda dar paso a la acogida y la ESI encuentre mayores posibilidades de concreción.

\section{Referencias bibliográficas}

Argentina, Ministerio de Educación de la Nación. (2008). Lineamientos Curriculares para la Educación Sexual Integral. Buenos Aires: Argentina.

Argentina, Ministerio de Educación de la Nación. (2012). Educación Sexual Integral para la Educación Secundaria II: contenidos y propuestas para el aula. Buenos Aires: Argentina.

Argentina. Programa Nacional de Educación Sexual Integral. Ley N.²6.150. Sancionada en 4 de octubre de 2006.

Butler, J. (2001). El género en disputa. El feminismo y la subversión de la identidad. Barcelona: Paidós.

Bracht, V. \& Crisonio, R. (coord.) (2003). La educación física en Argentina y en Brasil: Identidad, desafíos y perspectivas. La Plata: Al margen.

Fernández, M. L. (2011). Los estereotipos de género en las clases de Educación física en el nivel medio [Tesis de Licenciatura]. Recuperada el 30 de julio de 2019, de http://imgbiblio.vaneduc.edu.ar/fulltext/files/TC104960.pdf

Bracht, V. (1996). Educación Física y aprendizaje social. Educación Física/ciencia del deporte: ¿Qué ciencia es esa? Córdoba: Editorial Vélez Sarsfield.

Colectivo Mariposas Mirabal. (2019). Cuadernos del IICE No 3. Educación Sexual Integral. Epistemología, pedagogía y política en los debates curriculares. Editorial de la Facultad de Filosofía y Letras. Recuperado el 16 de febrero de 2020, de http:/ /publicaciones.filo.uba.ar/sites/publicaciones.filo.uba.ar/files/ Cuaderno\%20IICE\%203.pdf

Córdoba, Argentina. Ministerio de Educación de la provincia de Córdoba. (2011). Diseño curricular del ciclo básico de la educación secundaria 2011-2015. Recuperado el 18 de octubre de 2019, de http://www.igualdadycalidadcba.gov.ar/SIPEC.CBA/publicaciones/EducacionSecundaria/LISTO \% 20 PDF/TOMO $\% 202 \%$ 20Ciclo\%20Basico\%20de\% 20la\%20Educacion\%20Secundaria\%20web\%208-211.pdf.

De Lauretis, T. (1996). La tecnología del género. Revista Mora, 2, 6-34. 
Di Gregorio, M. J. (2013). ¿Qué enseñamos en Educación Física en relación al género y la sexualidad?: Una propuesta de integración en la educación secundaria. Recuperado el 10 de septiembre de 2019, de http:// www. memoria.fahce.unlp.edu.ar/library? $a=d \& c=$ eventos $\& d=$ Jev3199.edu.ar/ fulltext/files/TC104960.pdf

Giordan, A. \& De Vecchi, G. (1988). Los orígenes del saber. Sevilla: Diada.

Kirk, D. (1990). Educación Física y Currículum. Introducción crítica. Valencia: Universitat de Valencia.

Kopelovich, P. (2017). Educación física y escuela secundaria. Sentidos y prácticas en torno a la enseñanza de la asignatura en un colegio de la provincia de Buenos Aires, Argentina [Tesis de Maestría]. Recuperada el 10 de octubre de 2019, de https://repositorio.flacsoandes.edu.ec/bitstream/10469/13729/2/TFLACSO2017PK.pdf

Kopelovich, P. \& Pansa, G. (2017). Educación Física, géneros y sexualidades: Hacia un estado de la cuestión en Argentina. Recuperado el 10 de marzo de 2019 de: http://sedici.unlp.edu.ar/handle/10915/75710.

Lamas, M. (1996). Usos, dificultades y posibilidades de la categoría 'género'. En M. Lamas (Comp.), El género: la construcción cultural de la diferencia sexual (pp. 265-302). PUEG/UNAM y Porrúa.

Marina, M., Garibaldi, M., Martín, P., Montes, M., Bilder, P., Nimo, M. et al. (2014). Clase 2: Los ejes de la ESI. Educación Sexual Integral. Especialización docente de nivel superior en educación y TIC. Buenos Aires: Ministerio de Educación de la Nación. Recuperado el 12 de marzo de 2020 de: http:// www.igualdadycalidadcba.gov.ar/SIPEC-CBA/Capacitacion 2016 / DocumentosSecundaria/Preceptores/Precep-Profu-EjesDeLaESI.pdf

Marozzi, J., Bosio, M. V. \& Bertarelli, P. (2018). La clase mixta en Educación Física: explorando obstáculos para su implementación. Investiga $+, 1,60-63$.

Martínez, L. \& Gómez, R. (2009). (coords.) La educación física y el deporte en la edad escolar. El giro reflexivo en la enseñanza. Buenos Aires: Miño y Dávila.

Morgade, G. \& Alonso, G. (2008). Cuerpos y sexualidades en la escuela: de la normalidad a la disidencia. Buenos Aires: Paidós.

Organización Panamericana de la Salud, Organización Mundial de la Salud (2000). Promoción de la salud sexual. Recomendaciones para la acción. Guatemala. Recuperado el 17 de marzo de 2020, de https://iris.paho.org/bitstream/handle/10665.2/ 51672/ReunionSaludSexual2000_spa.pdf?sequence $=1$ \&isAllowed $=y$.

Pellegrini Malpiedi, M. (2015). Pedagogía y educación física: prescripciones para un cuerpo hegemónico. Recuperado el 26 de marzo de 2019, de http:// rephip.unr.edu.ar/xmlui/handle/2133/5429.

Scharagrodsky, P. (1999). La problemática corporal en la educación física: los modelos corporales socialmente establecidos. Actas de investigación educacional: segunda jornada, Año II. La Plata: Universidad Nacional de La Plata. Recuperado 
el 13 de abril de 2019, de http://www.memoria.fahce.unlp.edu.ar/ library $? \mathrm{a}=\mathrm{d} \& \mathrm{c}=$ eventos $\& \mathrm{~d}=$ Jev7045.

Scharagrodsky P. (2004). Juntos pero no revueltos: la Educación Física mixta en clave de género. Cuadernos de Pesquisa, 34, 59-76.

Scharagrodsky, P. (2006). El padre de la Educación Física argentina: fabricando una política corporal generizada (1901-1938) En A. Aistenstein \& P. Scharagrodsky, Tras las huellas de la Educación física escolar argentina. Cuerpo, género y pedagogía: 1880-1950 (pp. 159-197). Buenos Aires: Prometeo.

Scharagrodsky, P. (2007). El cuerpo en la escuela. Explora pedagogía. Buenos Aires: Ministerio de Educación, Ciencia y Tecnología de la Nación.

Saraví, J. (1995). Hacia una educación física no sexista. Educación Física y Ciencia, $1,1-12$. 